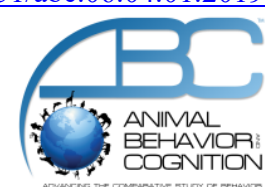

\title{
Animal Metacognition: A Decade of Progress, Problems, and the Development of New Prospects
}

\begin{abstract}
Michael J. Beran
Department of Psychology, Georgia State University

${ }^{*}$ Corresponding author (Email: mberan1@gsu.edu)

Citation - Beran, M. J. (2019). Animal metacognition: A decade of progress, problems, and the development of new prospects. Animal Behavior and Cognition, 6(4), 223-229. https://doi.org/10.26451/abc.06.04.01.2019

Abstract - In 2009, a special issue of Comparative Cognition and Behavior Reviews offered a series of target articles by researchers and theorists who debated the claim that animals were capable of engaging in metacognition. That special issue outlined some of the crucial points of contention at that time, and it also pointed the way toward refinements in the approaches used in empirical work with animals. The papers in that special issue framed the debate about whether animals' performances were better accounted for by learning mechanisms or first-order cognitive processes rather than metacognitive processes. In the decade since that special issue, substantial empirical and theoretical work has emerged on this question. This issue of Animal Behavior and Cognition presents some of that ongoing research and the new debate that it has generated. Proponents for and against claims that animals engage in metacognition offer their perspectives on existing data and what future research is needed to continue to move forward the exploration of animal metacognition. Research of the past decade has demonstrated a range of behavioral phenomena that cannot be accounted for by simply appealing to associative learning or monitoring external stimuli that serve as cues to evoke escape responses and other forms of metacognitive behaviors. However, there remain some important empirical and theoretical challenges to accepting that some animals are metacognitive (as was true also in 2009). These challenges should help frame the next decade of research, while at the same time being responsive to the full range of reported competencies shown by animals to date, and into the future.
\end{abstract}

Keywords - Metacognition, Comparative psychology, Comparative cognition, Control, Monitoring

In 2009, Comparative Cognition and Behavior Reviews hosted a special issue on animal metacognition. There were four target articles. Smith, Beran, Couchman, Coutinho, and Boomer (2009) acknowledged early results in this area were potentially explainable by low-level associative mechanisms that could create the appearance of uncertainty monitoring in animals (e.g., Smith, Beran, Couchman, \& Coutinho, 2008). However, Smith et al. (2009) also stated that not all experimental approaches were susceptible to such alternate explanations, and they argued that the next step in this area would be to better assess the cognitive-decisional performances that animals show rather than continue to engage in efforts to discount associative mechanisms. Specifically, they argued that researchers should be examining what kind of controlled processing animals could engage in, and whether those processes were executive, explicit, and declarative. Crystal and Foote (2009) took a different perspective, namely that most available data at the time were still interpretable from an associative, non-metacognitive perspective. They argued that better methods were needed, and that until there were more convincing data, a parsimonious conclusion was that most studies reflected the use of primary representations (or first-order states) rather than secondary representations (metacognitive, second-order states).

Another argument against claims for metacognitive animals was offered by Jozefowiez, Staddon, and Cerutti (2009) who reported that a simple behavioral model could account for the data produced by 
the dominant testing paradigms of that time (memory monitoring and uncertainty monitoring tasks). They suggested that it would be more fruitful to stop looking for metacognition in animals by adjusting the necessary criteria for that construct, and instead try to determine what theoretical models could already account for the behaviors the animals produced in the tests that were given to them. They noted some of the recent work (back in 2009) might challenge their behavioral model (e.g., the task described in Kornell, Son, \& Terrace, 2007), but even if it failed to account for those data, this would not strengthen a claim for metacognition in animals. Hampton (2009) proceeded to offer exactly the kind of revised criteria that Jozefowiecz et al. (2009) argued against. Because they have been relied upon so heavily in the decade since he offered them, they are re-presented here (Hampton, 2009, pp. 17-18):

1) We must specify a primary, objectively observable behavior that can be scored for accuracy or efficiency. Accuracy might be assessed as questions answered correctly, while efficiency could be assessed as time taken to learn all the assigned material.

2) There must be variation in the accuracy or efficiency of the primary behavior. Variation in performance is necessary in order to allow assessment of the correlation between the primary behavior and the secondary behavior (described in 3, below).

3) We must specify a secondary, objectively observable behavior that can be used to infer monitoring or regulation of cognition underlying the primary behavior. Monitoring of knowledge might be indicated by skipping questions for which the subject is unsure of the answer, while regulation might be indicated by subjects adjusting time spent studying to match the difficulty of the material.

4) There must be an explicit assessment of whether the primary and secondary behaviors are correlated. For example, were the questions that the subject skipped indeed ones for which he did not know the answer? Was study time adjusted appropriately to increase efficiency of learning? This correlation can be assessed most powerfully when the subject's knowledge is experimentally manipulated and their knowledge state can therefore be confidently known. For example, do subjects skip questions relating to information that has not been provided during training, while answering questions for which they have been trained on the correct answer?

These criteria have been adopted by many investigators since 2009 and will be referenced in some of the papers in this special issue of Animal Behavior and Cognition. And, the perspectives offered by Smith et al. (2009), Hampton (2009), Crystal and Foote (2009) and Jozefowiez et al. (2009) also will continue to be "felt" as the reader learns about the empirical work and the theoretical perspectives that work has generated since 2009. In some cases, opinions have changed. In others, they have not. At the risk of sounding self-congratulatory (given my own contributions to the area), I do think it is safe to say that nearly all interested parties have found the past decade to be intellectually stimulating, and in many cases to have provided real insights. In some ways, consensus has emerged that at least some of the experimental paradigms are now free from low-level, non-cognitive interpretations. Most interested parties would agree that executive processes may be engaged when some animals perform the tasks presented to them, although it is at this level that differences of opinion emerge as to whether such executive decision-making is likely to reflect metacognition or not.

\section{Progress: Expanded Evidence for Metacognition in Primate and Non-Primate Species}

In 2009, there was general acceptance that nonhuman primates, and especially great apes and rhesus monkeys, produced consistent behavioral patterns that could reflect metacognitive control and monitoring processes (see Call \& Carpenter, 2001; Hampton 2001; Kornell et al., 2007; Smith, Shields, \& Washburn, 2003). Since 2009, this evidence has greatly increased, as described in this issue by Hampton (2019). Hampton discusses that future work is needed to determine what information is available for metacognitive judgements at different phases of testing or training during experiments. This will enhance our understanding of cognitive processing generally, and metacognition specifically. Smith, Jackson, and Church (2019) suggest that researchers should turn more toward illuminating the cognitive 
architecture of capacities like metacognition. They argue that the question of whether nonhuman primates (and other animals such as dolphins) are metacognitive has been answered, affirmatively, and now it is time to move to questions about the structure of metacognitive systems in animals and what those might tell us about declarative awareness that is possibly present in animals' minds.

For other highly studied laboratory animals such as rats and pigeons, the evidence for metacognition in those species was equivocal in 2009. Although pigeons had been given a number of tests for metacognitive performance (Inman \& Shettleworth, 1999; Roberts et al., 2009; Sole, Shettleworth, \& Bennett, 2003; Sutton \& Shettleworth, 2008), there was inconsistent evidence of the patterns one would expect if pigeons could engage in metacognition. Since 2009, new reports offer stronger evidence. For example, Castro and Wasserman (2012) reported that pigeons learned to use and transfer use of a response that provided more information during discrimination tests. This suggested they were monitoring trial difficulty and using that response to make hard trials easier. Adams and Santi (2011) reported that pigeons demonstrated better performance for chosen memory tests than for forced memory tests in a duration matching-to-sample task, suggesting some commonalities with performance reported for monkeys. Iwasaki, Watanabe, and Fujita (2013) also reported that pigeons showed a greater likelihood for requesting "hints" on early trials when they were still learning sequences of responses than later when they had more experience with making those responses. In the present issue, Iwasaki, Kuroshima, and Fujita (2019) summarize their most recent work (Iwasaki Watanabe, \& Fujita, 2018) that shows even more sophisticated forms of metamemory that is engaged based on differential contributions of reference memory states or knowledge states. Importantly, they discuss the relation of working memory capacity on metacognition, and how load tasks could be an important avenue for research in this area (see also Smith, Coutinho, Church, \& Beran, 2013, for a related assessment of cognitive load and metacognition in monkeys).

In 2009, the case for metacognition in rats also was complicated. The bulk of the research was conducted by Crystal, who initially advocated for a metacognitive interpretation of rat behavior during tests in which rats showed they would decline difficult trials more often than easy trials (Foote \& Crystal, 2007). However, Crystal and Foote (2009) discussed those data very differently in light of the claims made by Smith et al. (2008) that one could model those performances without need of invoking metacognitive processes. In this issue, Crystal (2019) outlines his thinking and how it changed based on the article by Smith et al. (2008) and his reconsideration of data from rats and other species (but also see Foote \& Crystal, 2012). Crystal (2019) remains skeptical that current empirical work is sufficiently clear on the need to invoke metacognition to explain what animals do in the tasks presented to them. He offers that the general-purpose non-metacognitive models proposed by Smith et al. (2008) and others (e.g., Le Pelley, 2012) should be used as crucial tests of claims offered as evidence of metacognition in animals. He also advocates for explicit identification of the stages of information processing that could be proposed as evidence of genuine metacognition. Computational modeling is one way to do this, by comparing metacognition and non-metacognition models, and, from that, establishing new experimental procedures that could be explained only by metacognition (without also being explained by a nonmetacognition model). Yuki, Sakurai, and Okanoya (2019) propose in this issue that another way to distinguish whether metacognition is at work in the performance of animals is to understand and carefully manipulate the degree to which animals must respond to internal cues and come to rely on those cues to guide behavior. They review recent work from their laboratory (Yuki \& Okanoya, 2017) to demonstrate how this approach can be beneficial not only for studying animal metacognition, but also human metacognition (e.g., Yuki, Nakatani, Nakai, Okanoya, \& Tachibana, 2019). Finally, in this issue, Templer (2019) summarizes recent work from her laboratory (Templer, Lee, \& Preston, 2017) that shows that the right approach, that takes into account some of the natural tendencies of the species under study, can produce stronger evidence of metacognitive patterns of behavior. And, Templer (2019) notes that this is an important development because rats are a widely used model for understanding neurobiology and its relation to behavior. Thus, studies with rats could produce a clearer picture of the relation of brains and behavior for metacognition. 


\section{Problems: The Continuing Debate - Is it Metacognition?}

The major point of contention in this area of research in 2009 was whether the responses that were made by some animals to tests of varying difficulty or to varying states of knowledge about a given trial were best accounted for by metacognitive processes or associative learning processes. That debate has subsided for the most part, although as I noted above Crystal (2019) argues that there is still caution needed regarding this issue. There is now at least a stronger consensus than in 2009 that the performances seen in many tests of animal metacognition are not conditioned by external cues and are not the result of associative learning only. The criteria set by Hampton (2009) often are met, and the resulting performances can be argued to reflect executive processes. This idea is distributed throughout many of the articles in this special issue.

What is now contested is whether these descriptions of controlled, executive processes that underlie primary task performance and the monitoring of that task performance should be claimed as evidence of metacognition in animals. Carruthers and Williams (2019) argue that they should not. They claim that comparative psychologists are still prematurely concluding that metacognition must be occurring in these tests. They examine the uncertainty monitoring task (Smith et al., 2003) and the information-seeking tasks (Call \& Carpenter, 2001) in detail, and they conclude that the first type of task is really about first-order estimates of risk whereas the second is about first-order states of curiosity. This is not sufficient to claim metacognition in animals (see Carruthers, 2008, 2014). Despite arguing this, Carruthers and Williams also demonstrate the shift from earlier concerns about associative learning to current concerns about first-order and higher-order cognition by noting that "...that the root of our objections to the primate-metacognition literature is not that we think the data are better explained in mere associative terms. On the contrary, we fully accept that these (and many other) animals are capable of flexible executively-controlled decision making" (p. 279). Carruthers and Williams (2019) also describe their own recent study with human participants that showed there was little or no relation within these participants regarding performance on an uncertainty monitoring task and a declarative test in which confidence judgments were pronounced by participants (Nicholson, Williams, Grainger, Lind, \& Carruthers, 2019). Autism spectrum disorder participants performed equivalently to neurotypical participants on the uncertainty task, but not on the confidence judgment task where they were more impaired. This was offered as an argument against uncertainty monitoring tasks as measures of metacognition. Interestingly, performance on the confidence judgment task was related to mindreading abilities, a result that led Carruthers and Williams to state that the "findings support the view that explicit forms of metacognition employ some of the same cognitive resources as mindreading, and that implicit tasks of the sort conducted with monkeys are not genuinely metacognitive in nature" (p. 281). This issue of implicit versus explicit metacognition also is discussed at length in this special issue by Comstock (2019). He argues that confusion occurs around the idea of "implicit" metacognition, which is unlikely to reflect what philosophers mean by metacognition. Instead, he argues that studies of implicit metacognition are better described as studies of cognition alone. This gets at the heart of the ongoing debate. Can one be engaging in metacognition without knowing one is in that state of reflecting on knowledge or gauging certainty or uncertainty? Carruthers and Williams would argue that the answer is No. Comstock agrees and argues that metacognition and consciousness are not dissociable, and that we should be defining conscious metacognition so as to then move forward in our empirical efforts to see whether animals are, in fact, metacognitive. Neither Carruthers and Williams or Comstock deny the possibility that animals are metacognitive, but they deny that the existing data point to that conclusion. They believe that the existing data cannot be used to argue for declarative, explicit, second-order processes that, for them, are central to the definition of metacognition. This last point is an important one, because comparative researchers often argue that metacognition does not require declarative, conscious awareness to manifest, and does not require the capacity for mindreading or metarepresentational abilities (e.g., Couchman, Coutinho, Beran, \& Smith, 2009; Hampton 2019, this issue; Marsh, 2019, this issue; Smith et al., 2019, this issue). And, as Proust (2019) outlines in this issue, not all philosophers would define metacognition in the way that Carruthers and Williams or Comstock choose to define it. Proust 
discusses research with nonhuman animals and with children, and she takes a more inclusive perspective on whether such studies demonstrate metacognitive abilities in animals and children. Her argument is that developmental, neuroscientific and comparative studies all indicate that cognitive agents can pursue informational goals and reliably monitor them. This is a point of agreement with Carruthers and Williams and Comstock. However, she claims that although it may be that children and animals do this without representing their own mental states as mental states, this is still a form of "procedural" metacognition that is different from first-order cognitive processes. She then outlines and discusses some of the objections that have been raised about comparative and developmental research, providing her responses to those objections.

\section{Prospects: What to Expect in the Next Decade}

Comstock (2019) in this issue provides a broad assessment of past and proposed future work that he thinks is essential to move this area forward. He states that the field has progressed past questions of associative-only explanations, and he argues that what is needed now is a broader effort at the behavioral, neurobiological, and genetic levels to provide (if they exist) converging lines of evidence to support a conclusion that animals are consciously metacognitive. This is a forward-looking perspective that may help guide future research, although there will certainly be discussion about his proposed requirements to conclude that metacognition is present. Other contributors to the special issue also argue that it is time to take new empirical approaches. Marsh (2019) states that we can be more precise in determining exactly how animals consider that information that they can access and how they distinguish that information from information that still may be needed. Her previous research (e.g., Marsh \& MacDonald, 2012) showed that orangutans do engage in the information-seeking behaviors that suggest awareness of states of knowing and not knowing (also Beran, Smith, \& Perdue, 2013; Call, 2010; Call \& Carpenter, 2001). Now, she advocates asking about what types of information animals will gather and how they know how to gain information. And, she argues that more can be done to understand how animals modify their search behavior depending on how informative the source is. This could require an intermediate step toward the question of mindreading, in some cases, when other individuals may be the sources of information.

Ferrigno, Bueno, and Cantlon (2019) correctly note that there are situations in which humans do not rely on introspection to make metacognitive judgments but instead rely on heuristic strategies based on perceptual salience. What is fascinating in these cases is that such heuristics are themselves fallible, setting the stage for situations in which the control and monitoring functions of a metacognitive system may fall prey to biases or illusions that impact their operation. In other words, this approach allows for errors and failures to actually reflect metacognition in the fallible individual. Ferrigno, Kornell, and Cantlon (2017) showed that monkeys also demonstrate fallible confidence judgments that are based on salient perceptual features of the stimuli rather than objective performance levels in discrimination and memory tests. This is a very important result, because it moves away from studying metacognition in animals only as a means of showing their ability to efficiently deal with difficulty or ignorance, and instead demonstrates a key aspect of human metacognition which is the self-evaluation of internal cues that may, themselves, be inaccurate reflections of reality (see Kornell, 2014). This is an exciting idea for future research, to examine metacognitive errors in comparative perspective, and combined with the proposals for future research directions by Marsh, Comstock, Smith et al., and other contributors to this special issue it is easy to imagine that the next decade will reflect empirical work that is responsive to these proposals.

Schwartz (2019) concludes the special issue by offering another novel perspective to the question of animal metacognition, based on an evolutionary lens. He notes that nonhuman primates are crucial subjects for understanding human cognitive evolution, and perhaps also human metacognitive evolution. But his prescription for the field is to move more toward questions of ecological significance for the animals being studied, given that ecology certainly influenced the evolution of human cognition. He notes that a benefit of this is also the avoidance of assessing animal cognition (and metacognition) 
only to see if it is like human cognition (and metacognition). In many ways, this prescription accommodates many of the points of contention about what animal metacognition should look like, if it exists, and whether we should care that it is not identical to human metacognition. Schwartz tells us that there is great value in understanding how different ecological niches could generate different routes to cognitive and potentially metacognitive capacities that may help explain the data we see in our comparative studies. This perspective also offers important guidance for what may be the next decade of research in this field. Perhaps in 10 more years we may have radically different views of animal metacognition given the guidance offered by all authors in this special issue (and also the excellent efforts of those who are not included here but who are actively working in the area). It is almost a certainty that the pursuit of new data and how to interpret those data will continue to be a major focus in comparative cognition.

\section{References}

Adams, A., \& Santi, A. (2011). Pigeons exhibit higher accuracy for chosen memory tests than for forced memory tests in duration matching-to-sample. Learning \& Behavior, 39, 1-11.

Beran, M. J., Smith, J. D., \& Perdue, B. M. (2013). Language-trained chimpanzees name what they have seen, but look first at what they have not seen. Psychological Science, 24, 660-666.

Call, J. (2010). Do apes know that they could be wrong? Animal Cognition, 13, 689-700.

Call, J., \& Carpenter, M. (2001). Do apes and children know what they have seen? Animal Cognition, 4, 207-220.

Carruthers, P. (2008). Meta-cognition in animals: A skeptical look. Mind \& Language, 23, 58-89.

Carruthers, P. (2014). Two concepts of metacognition. Journal of Comparative Psychology, 128, 138-139.

Carruthers, P., \& Williams, D. M. (2019). Comparative metacognition. Animal Behavior and Cognition, 6, 278-288.

Castro, L., \& Wasserman, E. A. (2013). Information-seeking behavior: Exploring metacognitive control in pigeons. Animal Cognition, 16, 241-254.

Comstock, G. (2019). What do we need to know to know that animals are conscious of what they know? Animal Behavior and Cognition, 6, 289-308.

Couchman, J. J., Countinho, M. V. C., Beran, M. J., \& Smith, J. D. (2009). Metacognition is prior. Behavioral and Brain Sciences, 32, 142.

Crystal, J. D. (2019). Comparative approaches to metacognition: Prospects, problems, and the future. Animal Behavior and Cognition, 6, 254-261.

Crystal, J. D., \& Foote, A. L. (2009). Metacognition in animals. Comparative Cognition \& Behavior Reviews, 4, $1-$ 16.

Ferrigno, S., Bueno, G., \& Cantlon, J. F. (2019). A similar basis for judging confidence in monkeys and humans. Animal Behavior and Cognition, 6, 335-343.

Ferrigno, S., Kornell, N., \& Cantlon, J. F. (2017). A metacognitive illusion in monkeys. Proceedings of the Royal Society B: Biological Sciences, 284, 20171541.

Foote, A. L., \& Crystal, J. D. (2007). Metacognition in the rat. Current Biology, 17, 551-555.

Foote, A. L., \& Crystal, J. D. (2012). "Play it again": A new method for testing metacognition in animals. Animal Cognition, 15, 187-199.

Hampton, R. R. (2001). Rhesus monkeys know when they remember. Proceedings of the National Academy of Sciences of the United States of America, 98, 5359-5362.

Hampton, R. R. (2009). Multiple demonstrations of metacognition in nonhumans: Converging evidence or multiple mechanisms? Comparative Cognition \& Behavior Reviews, 4, 17-28.

Hampton, R. R. (2019). Monkey metacognition could generate more insight. Animal Behavior and Cognition, 6, 230-235.

Inman, A., \& Shettleworth, S. J. (1999). Detecting metamemory in nonverbal subjects: A test with pigeons. Journal of Experimental Psychology: Animal Behavior Processes, 25, 389-395.

Iwasaki, S., Kuroshima, H., \& Fujita, K. (2019). Pigeons show metamemory by requesting reduced working memory loads. Animal Behavior and Cognition, 6, 247-253.

Iwasaki, S., Watanabe, S., \& Fujita, K. (2013). Do pigeons (Columba livia) seek information when they have insufficient knowledge? Animal Cognition, 16, 211-221.

Iwasaki, S., Watanabe, S., \& Fujita, K. (2018). Pigeons (Columba livia) know when they will need hints: Prospective metacognition for reference memory? Animal Cognition, 21, 207-217.

Jozefowiez, J., Staddon, J. E. R., \& Cerutti, D. T. (2009). Metacognition in animals: How do we know that they know? Comparative Cognition \& Behavior Reviews, 4, 29-39. 
Kornell, N. (2014). Where is the "meta" in animal metacognition? Journal of Comparative Psychology, 128, 143149.

Kornell, N., Son, L. K., \& Terrace, H. S. (2007). Transfer of metacognitive skills and hint seeking in monkeys. Psychological Science, 18, 64-71.

Le Pelley, M. E. (2012). Metacognitive monkeys or associative animals? Simple reinforcement learning explains uncertainty in nonhuman animals. Journal of Experimental Psychology: Learning, Memory, and Cognition, $38,686-708$.

Marsh, H. L. (2019). The information-seeking paradigm: Moving beyond 'if and when' to 'what, where, and how.' Animal Behavior and Cognition, 6, 329-334.

Marsh, H. L., \& MacDonald, S. E. (2012). Orangutans (Pongo abelii) "play the odds": Information-seeking strategies in relation to cost, risk, and benefit. Journal of Comparative Psychology, 126, 263-278.

Nicholson, T., Williams, D.M., Grainger, C., Lind, S., \& Carruthers, P. (2019). Relationships between implicit and explicit uncertainty monitoring and mindreading: Evidence from autism spectrum disorder. Consciousness and Cognition, 70, 11-24.

Proust, J. (2019). From comparative studies to interdisciplinary research on metacognition. Animal Behavior and Cognition, 6, 309-328.

Roberts, W. A., Feeney, M. C., McMillan, N., MacPherson, K., Musolino, E., \& Petter, M. (2009). Do pigeons (Columba livia) study for a test? Journal of Experimental Psychology: Animal Behavior Processes, 35, 129-142.

Schwartz, B. L. (2019). Using natural ecology to predict higher cognition in human and non-human primates. Animal Behavior and Cognition, 6, 344-354.

Smith, J. D., Beran, M. J., Couchman, J. J., \& Coutinho, M. V. C. (2008). The comparative study of metacognition: Sharper paradigms, safer inferences. Psychonomic Bulletin \& Review, 15, 679-691.

Smith, J. D., Beran, M. J., Couchman, J. J., Coutinho, M. V., \& Boomer, J. B. (2009). Animal metacognition: Problems and prospects. Comparative Cognition \& Behavior Reviews, 4, 40-53.

Smith, J. D., Coutinho, M. V. C., Church, B., \& Beran, M. J. (2013). Executive-attentional uncertainty responses by rhesus macaques (Macaca mulatta). Journal of Experimental Psychology: General, 142, 458-475.

Smith, J. D., Jackson, B. N., \& Church, B. A. (2019). The cognitive architecture of uncertainty. Animal Behavior and Cognition, 6, 236-246.

Smith, J. D., Shields, W. E., \& Washburn, D. A. (2003). The comparative psychology of uncertainty monitoring and metacognition. Behavioral and Brain Sciences, 26, 317-339.

Sole, L. M., Shettleworth, S. J., \& Bennett, P. J. (2003). Uncertainty in pigeons. Psychonomic Bulletin \& Review, $10,738-745$.

Sutton, J. E., \& Shettleworth, S. J. (2008). Memory without awareness: Pigeons do not show metamemory in delayed matching to sample. Journal of Experimental Psychology. Animal Behavior Processes, 34, 266282.

Templer, V. L. (2019). Slow progress with the most widely used animal model: Ten years of metacognition research in rats, 2009-2019. Animal Behavior and Cognition, 6, 273-277.

Templer, V. L., Lee, K. A., \& Preston, A. J. (2017). Rats know when they remember: Transfer of metacognitive responding across odor-based delayed match-to-sample tests. Animal Cognition, 20, 891-906.

Yuki, S., Sakurai, Y., \& Okanoya, K. (2019). The utility of internal cognitive states as discriminative cues affecting behavioral adaptation in humans and animals. Animal Behavior and Cognition, 6, 262-272.

Yuki, S., Nakatani, H., Nakai, T., Okanoya, K., \& Tachibana, R., O. (2019). Regulation of action selection based on metacognition in humans via a ventral and dorsal medial prefrontal cortical network. Cortex, 199, 336-349.

Yuki, S., \& Okanoya, K. (2017). Rats show adaptive choice in a metacognitive task with high uncertainty. Journal of Experimental Psychology: Animal Learning and Cognition, 43, 109-118. 